\section{Some Notes on Re-Embedding EM Samples}

Robert (Bob) Chiovetti, Microimaging Technologies, Inc.

Most of us have been here more than once: a critical specimen, often the only one of its kind, does not embed properly. Usually the problem is incompletely polymerized resin. After the panic subsides, a limited number of options are left, and if the specimen is irreplaceable, there are no options, other than trying to salvage it the best way you can. I've had this problem before with low viscosity resins, but the following suggestions should work for other resins as well.

First of all, realize that reprocessed specimens are rarely as good as they would be if things went fine the first time around. But when only a few sections are needed from a critical block, I would suggest trying the following, in this order:

1) Crank up the heat in the polymerizing oven, and really "cook" the specimen for an additional 12 to 24 hours. If the resin polymerizes around $70^{\circ} \mathrm{C}$, try repolymerizing the block at $90^{\circ}$ to $100^{\circ} \mathrm{C}$. This will frequently harden a marginal block enough to get at least a few sections from it.

2) If repolymerization doesn't work, try using propylene oxide to remove most of the gooey stuff. Use the binocular scope and the trimming block on the ultramicrotome to remove most of the resin from around the specimen. Use lots of razor blades, changing them frequently. I like to use the thinner, double-edge blades for this. Then cut under the specimen to remove it from the block. Be careful at this step. If too much force is used, the valued specimen will go flying across the room, never to be seen again.

It depends on how much crosslinking has taken place in the soft parts, but it should be possible to remove at least some of the soft resin with propylene oxide.

\section{Remove the Veil of Contamination from SEM Samples!}

\section{NEW!}

Flash SEM-CLEAN in-situ Cleaning

- Quickly remove hydrocarbon scum from SEM chamber walls and samples

- Clean samples in-situ

- Fast - less than 5 minutes

- Activated Oxygen cleaning action

- Safe - No sputter etching

- Ideal for Materials Science and Metrology

- No exotic gases - No Argon

- Patent pending

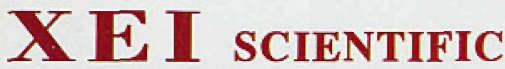

Anti-Contamination Systems for SEMs

3124 Wessex Way, Redwood City, CA 94061

(650) 369-0133, FAX (650) 363-1659

http:/www.msa.microscopy.com/SM/XEI/XEIHomePage.html
Place the unblocked specimen in a vial with a large excess of propylene oxide, and place the vial on a rotator or an agitator. Make frequent changes of propylene oxide over a period of 24 to 48 hours. From this point, it should be possible to infiltrate as usual. Try an ascending series of resin concentrations that are cut with propylene oxide, then pure resin overnight. Apply vacuum during the infiltration with pure resin if possible. Using a vacuum oven for polymerization is also a good idea.

The objective here is to adequately infiltrate and polymerize good, hard resin into the specimen as far as possible. The center of the specimen will probably still be soft, so when sectioning the re-embedded specimen, be sure to concentrate on collecting sections from the first few micrometers of the surface.

An interesting alternative (which I have not personally tried) might be to use sodium ethoxide instead of propylene oxide to remove resin from the specimen. Sodium ethoxide is extremely corrosive. A one minute incubation on a drop of sodium ethoxide will completely digest the resin from an $80 \mathrm{~nm}$ thin section, so it should work great to remove soft resin from a specimen. Sodium ethoxide is sodium hydroxide in ethanol, so dehydration with ethanol is needed following the treatment, then go into propylene oxide then infiltrate in the usual fashion. If anyone has tried this I would like to hear the results.

Maybe there's a grad student out there who is looking for a little experiment to do this weekend?

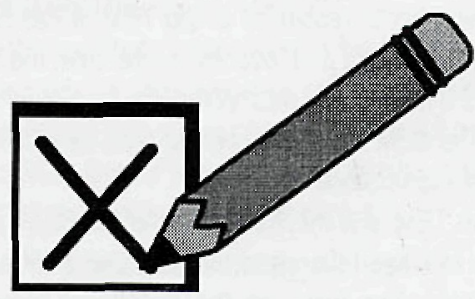

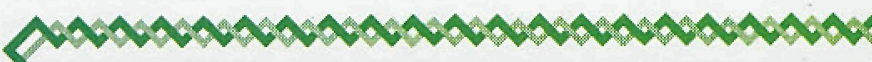 \\ WinEDS 3.0 High Performance X-ray Microanalysis For Windows ${ }^{\mathrm{TM}} 95$ / NT 4.0}

This FAST full 32-bit PC Windows ${ }^{\text {TM }}$ based analyzer gives you all the performance of more expensive workstation systems.

* Qualitative \& Quantitative software with one click operation.

* WinEDS PX9000 Pulse Processor DX3000 Bias.

* Interfaces to several detector brands.

* 5 to $10 \mathrm{eV}$ improvement on most detector's resolutions.

* Complete system upgrades - including new detectors.

* Partial upgrades to suit your system needs. Also

* Complete line of PC based imaging systems.

* Top dollar for trade-ins.

* Service for TN5500 and TN2000 systems.

TN Analyzer Service Inc. 7897 Hwy 19
Dane, WI $\mathbf{5 3 5 2 9}$

Tel.: (608)798-2005, Fax: (608)798-1675, eMail: doug_tnas@msn.com

Visit our web site: www.tnanalyzerservice.com Now with demo labs in Florida and Texas, as well as in Wisconsin - and soon on the west coast. 

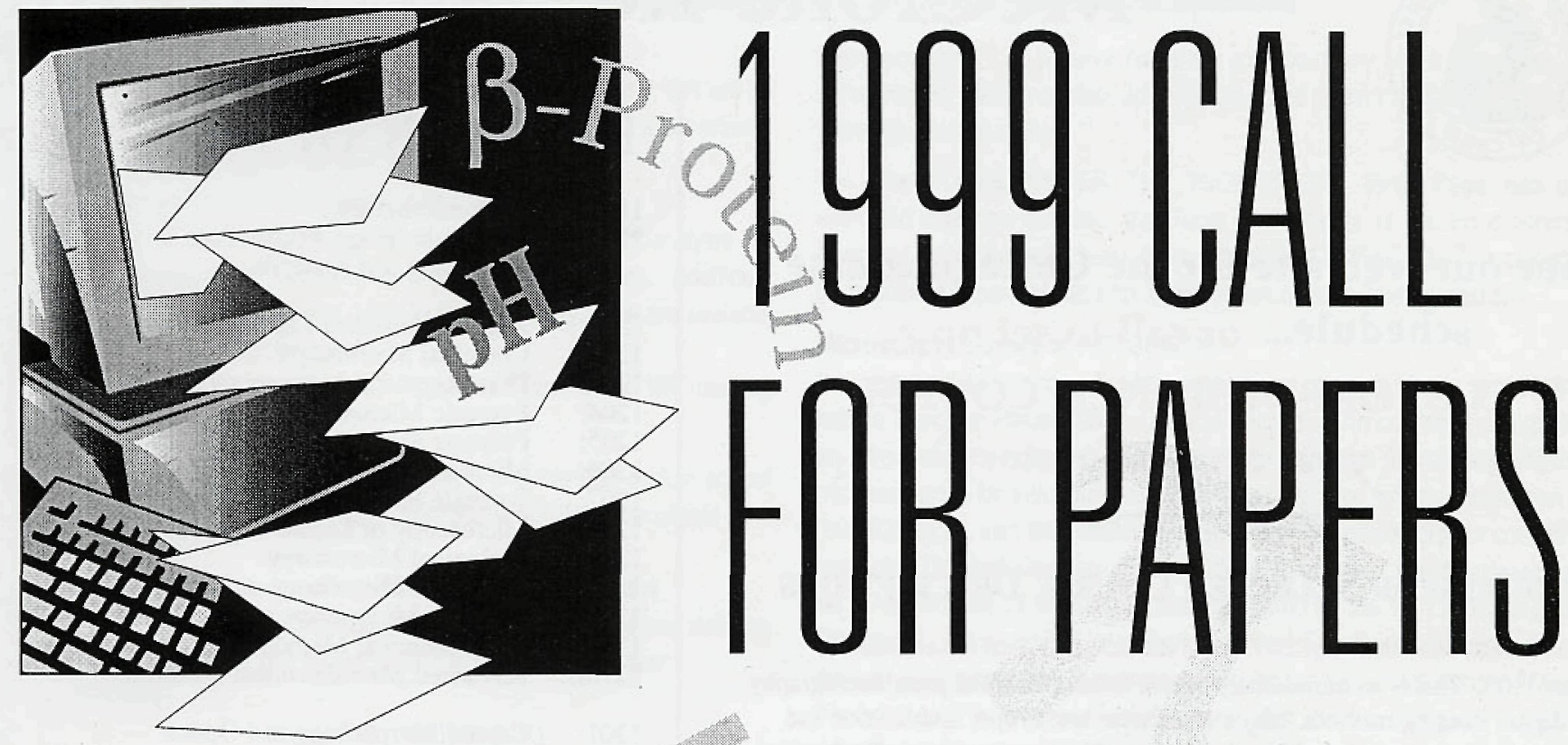

Please make the 1999 Eastern Analytical Symposium the best ever, by submitting your own paper for inclusion in the technical program. The Symposium will be held on Nov. 14-19, 1999. Abstracts of the proposed papers should be 200 to 250 words, please indicate your preference for an oral or poster format to the committee.

Send your submission to:

P.0. Box 633, Montchanin, DE 19710-0633 or send email to: easinfo@aol.com.

For more information visit our EAS web site: http://www.eas.org/

Use our online submission form.

The deadline for Submission

of preliminary abstracts

is April 16,1999.

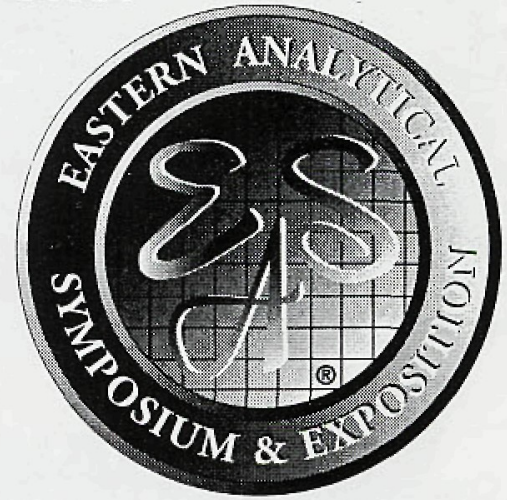

EAS Hotline:1-302-738-6218 • Faxline:1-302-738-5275 • email: easinfo@aol.com. 


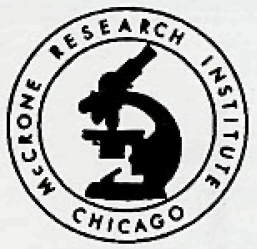

See our web site for the Chicago course schedule... or call to set up a CUSTOM-DESIGNED COURSE at your own laboratory.

\section{New and/or Selected Course Descriptions}

\section{Electronic Image Acquisition, Processing and Analysis -}

Course 1105. This is an introductory course linking classical photomicrography with digital imaging methods. Major topic areas are: Proper illumination and microscope adjustment; Assessing the quality of image acquisition; Evaluation of image acquisition hardware and software; Image storage; Methods of image processing and enhancement; Segmentation and thresholding operations; Measurements; and Evaluation of image display and printing hardware. The emphasis in this course is on the evaluation and control of one's imaging system in relation to one's own imaging requirements. Image processing is included as a portion of the course, but not as the primary emphasis.

SCANNING Electron Microscopy - Course 1402. This is a comprehensive, hands-on course on scanning electron microscopy. Included are the theory of SEM, design of instruments, sample preparation, trouble-shooting, qualitative and quantitative $x$-ray analysis and the quality of data and images. The goal is to acquire sufficient understanding of the instrument to effectively adapt to sample and instrument-related problems as they occur. Metals, polymers, biological materials and particles are among the materials examined. Students are encouraged to bring samples and problems for their individual projects.

Transmission Electron Microscopy of Materials - Coutse 1407. This course is intended for those with some working knowledge of electron beam instruments and a desire to become better equipped to carry out transmission electron microscopy and microanalytical studies in the materials sciences. Topics for lecture and laboratory work include specimen preparation, principles of imaging, image interpretation, selected area electron diffraction, $x$-ray microanalysis, electron energy loss spectroscopy, and image processing techniques. Laboratory exercises emphasize practical problem solving skills, image quality and interpretation of results.

The scope of the course is broad, with concentration on those topics of most interest to participants. Students are encouraged to bring their own specimens and problem samples to class in order to focus their efforts on the materials most relevant to them.

AdVANCED FTIR Microspectroscopy - Course 1422. This course is designed for the experienced FTIR microscope user, who wants to maximize instrument performance and learn specialized methods including sample handling, ATR, reflection/absorption, grazing angle techniques, mapping, hotstage methods and spectral interpretation. The integration of FTIR microspectroscopy and light microscopy is stressed.

\section{MicRosCOPY COURSES}

1101: Photomicrography

1105: Electronic Image Acquisition, Processing and Analysis

1201: Applied Polarized Light Microscopy

1202: Chemical Microscopy

1203: Pharmaceutical Microscopy

1204: Forensic Microscopy

1205: Polymer Microscopy

1206: Microscopy for Art Conservators

1207: Forensic Hair and Fiber Microscopy

1209: Microscopy of Liquid Crystals

1235: Biological Microscopy

1250: Advanced Microscopy and Special Methods

1260: Hotstage Microscopy and Polymorphism

1270A: Microchemical Methods

1270B: Advanced Microchemical Methods

1301: Crystal Morphology and Optics

1302: Advanced Crystallography

1310: Conoscopic Methods

1402: Scanning Electron Microscopy

1407: Transmission Electron Microscopy of Materials

1422: Advanced FTIR Microspectroscopy

1501A: Identification of Small Particles

1501B: Advanced Small Particle Identification

1501E: Particle Isolation, Manipulation and Mounting for Additional Analysis

1503: Mineral Grain Identification

1505: Microscopy and Microchemistry of Polymers

1507: Advanced Fiber Identification

1536: Microscopy of Botanical Traces

1537: Advanced Pollen and Spore Identification

1540: Wood and Vegetable Fiber Identification

1560: Food Microscopy

1608A: Microscopical Identification of Asbestos

1608B: Advanced Asbestos Identification

1612: Special Asbestos Problems

1616: Asbestos Fiber Counting (NIOSH 582)

1628: Quantitative Asbestos Analysis

1629: Advanced Asbestos QA/QC

1630: Indoor Air Quality: Microscopy of

Fungal Spores and Pollen

1640: Microscopy of Particulate Air Pollutants and Hazardous Settled Dust

1701: Advanced Forensic Microscopy (Trace Evidence)

1705: Forensic Hair Microscopy

1710: Comparative Microscopy of Soils

1715: Forensic Paint Microscopy

1717: Forensic Examination of Building Materials

1722: Microscopy of Explosives

1726: Microscopy of Illicit Drugs and Excipients

1750: Source Determination of Particle Samples

1801: Thesis Research

1901: Custom Planned On-Site Courses

\section{FOR ADDITIONAL INFORMATION}

Contact: Nancy Daerr or Alicia Jimenez at McCrone Research Institute, 2820 S. Michigan Avenue, Chicago, IL 60616-3292 